\title{
A system of gamma ray imaging devices with coded apertures
}

\author{
Christos Papadimitropoulos ${ }^{1}$, loannis Kaissas ${ }^{2}$, Konstantinos Karafasoulis ${ }^{3}$, Constantinos Potiriadis ${ }^{2}$ and Charalambos $\mathrm{P}$. \\ Lambropoulos ${ }^{1, a}$ \\ ${ }^{1}$ Technological Educational Institute of Sterea Ellada, 34400 Psahna, Evia, Greece \\ ${ }^{2}$ EDP Greek Atomic Energy Commission, 15310 Agia Paraskevi, Attiki, Greece \\ ${ }^{3}$ Hellenic Army Academy, 16673 Vari, Attiki, Greece
}

\begin{abstract}
We describe a system consisting of two CdTe based gamma cameras with coded apertures and a video camera. The system is used for the localization of radioactive sources with the aid of triangulation. The methods used and the performance of the system are analyzed.
\end{abstract}

\section{Introduction}

Coded apertures are an alternative way of producing good quality images of gamma ray emitting objects mainly due to their increased efficiency compared to pinholes and collimator systems. Their signal-to-noise ratio (SNR) and adjustable Field of View (FoV) advantages can be traded off to reduce exposure times and improve resolution.

Meanwhile, the progress in the $\mathrm{Cd}(\mathrm{Zn}) \mathrm{Te}$ hybrid detector technology has made feasible the construction of truly portable gamma ray imaging devices operating at room temperature with superb energy and spatial resolution. Such devices with coded apertures in front of them can extract the identity of the source due to their capability to do spectroscopy as well as its direction. This is a very attractive combination for safety-security[1], medical[2] and astrophysics[3] applications.

Along this direction, we have developed a setup consisting of two coded aperture gamma cameras and we have studied the capability of this system to find the $\mathrm{x}, \mathrm{y}, \mathrm{z}$ coordinates of a point-like radioactive source.

\section{System description}

The system (Fig. 1) has two position sensitive gamma ray detectors with coded apertures placed on top of them. A video camera is included in the system. Acquired data from the two position sensitive detectors are collected and formatted by a custom-made data acquisition card and send to a PC where the result of their processing is fused with the image provided by the video camera. Both gamma cameras are placed on an aluminum base whose defines the origin of the system reference frame (SRF). The spatial coordinates of the source are estimated with respect to this reference frame. The gamma cameras separation distance $d$ can be adjusted. A horizontal PMMA plate is used for the accurate positioning of the sample radioactive source.

Each position sensitive detector consists of 8 hybrids arranged in 4 rows $\times 2$ columns. The hybrids are CdTe pixel detectors with $350 \mathrm{um}$ pixel pitch and $1 \mathrm{~mm}$ thickness bump bonded to CMOS ASICs. The active area of each detector is $4.4 \times 4.4 \mathrm{~cm}^{2}$ and it has 16384 pixels. The system can record signals from $30 \mathrm{KeV}$ up to $250 \mathrm{KeV}$ with $1 \mathrm{KeV}$ step and $3-4 \mathrm{keV}$ energy resolution as reported in[4] and verified by measurements with ${ }^{241} \mathrm{Am}$, ${ }^{57} \mathrm{Co}$ and ${ }^{99 \mathrm{~m}} \mathrm{Tc}$.

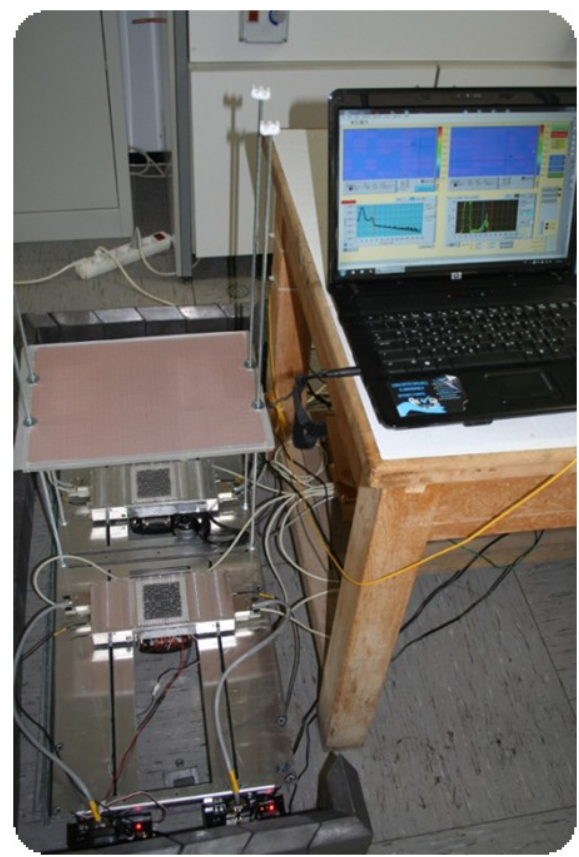

Figure 1. The experimental setup consists of two energy dispersive gamma ray counting devices, two coded aperture masks and a web camera. The setup is mounted on an aluminum base.

\footnotetext{
${ }^{\mathrm{a}}$ Corresponding author: lambrop@mail.teiste.gr
} 
The coded apertures used are Modified Uniform Redundant Arrays (MURA). Two pairs of MURA coded masks have been utilized. The one pair is of Rank 7 and the other of Rank 19. They have been constructed on a PMMA (Plexiglas) grid sheet with the following configuration: a) $5.6 \mathrm{~mm}$ holes in diameter and $3 \mathrm{~mm}$ pitch for Rank 7 and b) $2 \mathrm{~mm}$ holes in diameter and $1 \mathrm{~mm}$ pitch for Rank 19. The opaque elements of the Rank 7 masks are made of $2 \mathrm{~mm}$ thick $\mathrm{Pb}$ cylindrical blocks while the opaque elements of the Rank 19 mask are made of $1.9 \mathrm{~mm}$ thick $\mathrm{Pb}$ spheres.

Both the dimensions of the mask and the detector define a solid angle of operation which is known as Fully Coded Field of View (FCFOV). A radioactive source within the FCFOV illuminates the mask and projects a fraction of the mask pattern (shadowgram) onto the detector surface. Since two detectors are utilized, a system FCFOV (denoted as FCFOVS hereafter) is introduced as the common area of the FCFOV of the detectors and is directly related to both their separation $d$ and the mask-to-detector distance $z_{\text {mask }}$. Due to the detector housing, a minimum $z_{\text {mask }}=20 \mathrm{~mm}$ and $d=170 \mathrm{~mm}$ are introduced resulting to a minimum source to detector distance of $z_{\min }=69.4 \mathrm{~mm}$. Thus, for a point-like source positioned at $\mathrm{Z}>z_{\text {min }}$ the $F C F O V_{S}$ is $70 \times 70$ degrees.

\section{Reconstruction}

A radioactive source within the $\mathrm{FCFOV}_{\mathrm{S}}$ produces projected patterns (shadowgrams) on the detectors' surface (Fig. 2). These patterns are essentially a digital trail of the source on the position sensitive detector and

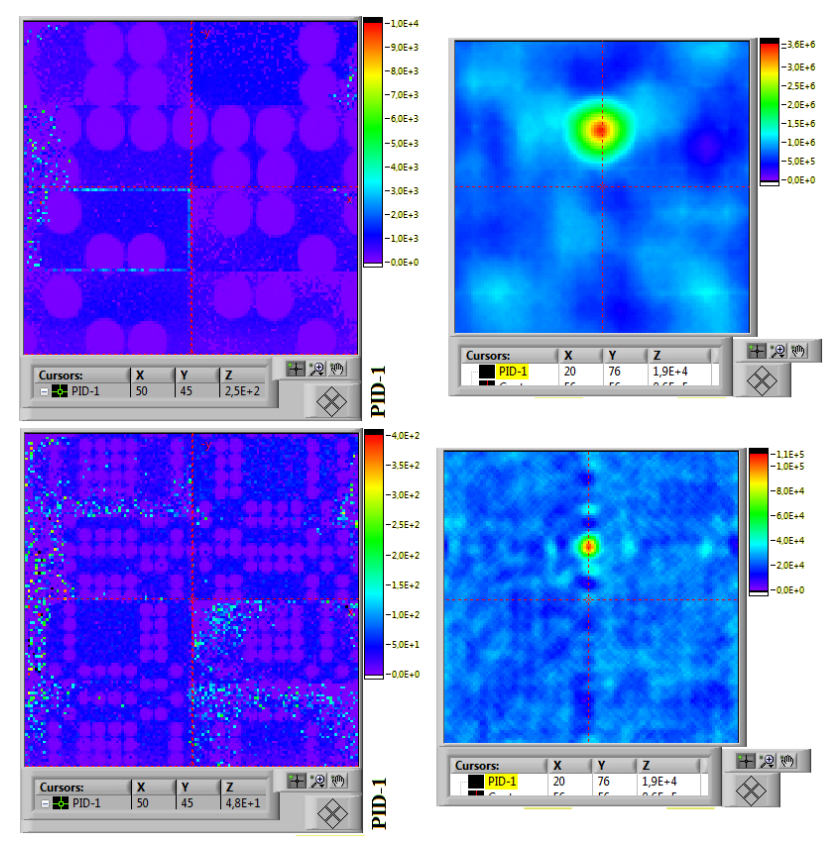

Figure 2. Experimental shadowgrams (left) on a detector surface and the reconstructed images (right) for Rank 7 (up) and Rank 19 (down), respectively for a point-like ${ }^{241} \mathrm{Am}$ source that illuminates both detectors. for this reason they can be used to infer the source direction. More specifically, the shadowgram is a modulated image of the source which is converted to the original image by cross-correlating it with a special decoding function, which is a replica of the mask pattern itself. The result is the so-called Source Point Spread Function (SPSF), a 2-D bell-shaped distribution (Fig. 3) [5]. The peak of the SPSF takes discrete adjacent positions as the source moves within the $\mathrm{FCFOV}_{\mathrm{S}}$, truncating the source location estimation. In order to overcome the unnecessary truncation error, the peak of the SPSF is fitted with a 2D Gaussian distribution, thus transforming the pixellated (digital) detector surface to a set of values for the spatial coordinates [6].

Since the system includes two gamma cameras, there are two SPSFs for every source location within the FCFOV $_{S}$. Thus, by using the peak of each SPSF and having the detector separation at hand, a triangulation algorithm can be applied to estimate the distance of the source along with its direction with respect to the SRF.

\section{Performance}

The reconstruction procedure presented in the previous section was performed using both experimental and simulated data. For the latter case, a pair of tools has been deployed: A fast-simulation code developed in the MATLAB programming environment [7] and a detailed simulation using the SWORD package [8]. Both simulations take into account all the parameters of the system, i.e. the pixel and the mask element size, the mask-to-detector distance, the separation between the detectors etc. Fig. 4 gives the estimated source

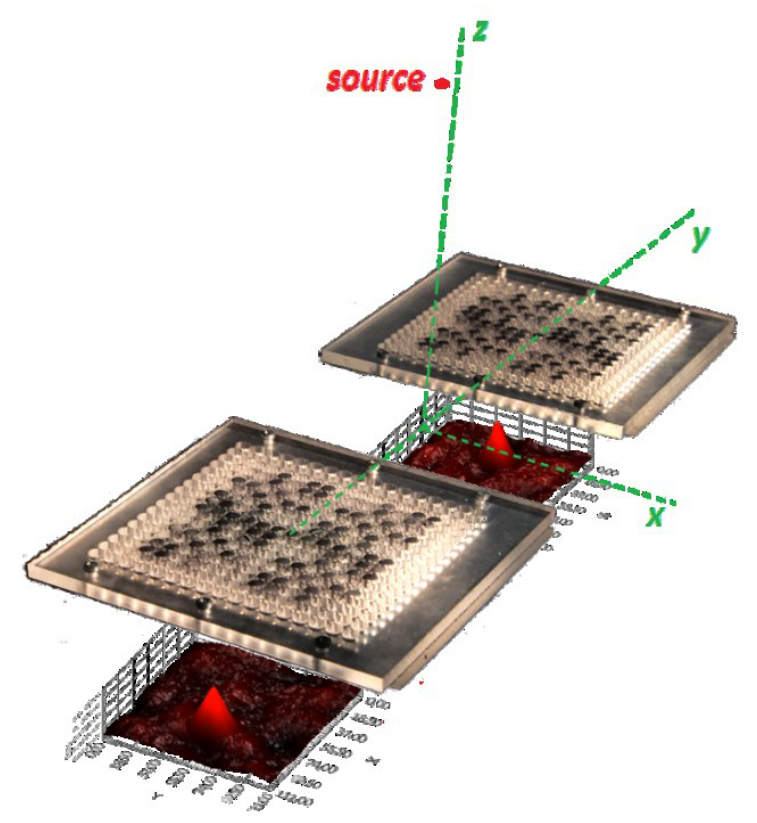

Figure 3. A schematic depiction of the triangulation method. The projected shadowgrams are cross-correlated with a decoding function producing a SPSF on each detector. The peaks of the SPSFs are inserted into the triangulation algorithm to estimate the source location. 
coordinates as a function of the real ones using the same reconstruction chain for real data and for simulation data produced with both fast and detailed simulation. The fair agreement between all the three derived trend lines verifies the reconstruction chain and the validity of the fast simulation.

The source location accuracy, i.e. the ability of the system to accurately estimate the position of a source within the FCFOV , is depicted in Fig. 5(a). A ${ }^{241} \mathrm{Am}$ source was placed at several locations on the XY plane $(\mathrm{Z}=150 \mathrm{~mm})$.

Another figure of merit of the instrument is its ability to separate two adjacent sources clearly. In Fig. 5(b-e), the shadowgrams along with the reconstructed images of two ${ }^{241} \mathrm{Am}$ sources in close proximity are presented for Ranks 7 (b,c) and 19 (d,e). These results demonstrate that the minimum distance of two sources located at $Z=150 \mathrm{~mm}$ is $48 \mathrm{~mm}$ and $32 \mathrm{~mm}$ for Ranks 7 \& 19 , respectively.
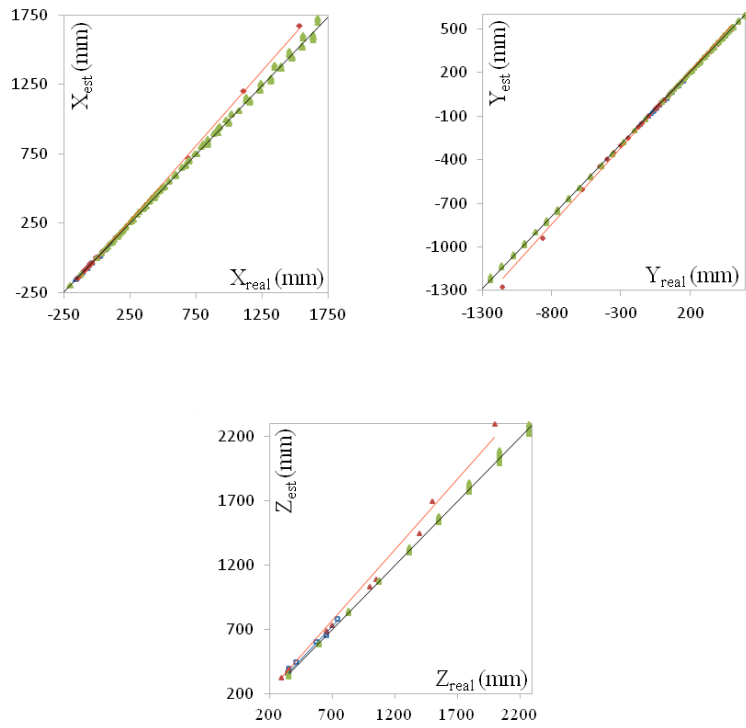

Figure 4. Comparison between the results of the reconstruction algorithm based on the experimental data (rhombs), simulated data produced by SWORD (squares) and simulated data produced by MATLAB (triangles).

\section{Conclusions}

The discussion in the previous sections has illustrated the performance of the system for the localization of pointlike sources. The analysis showed that the performance is enhanced when higher rank masks are utilized.
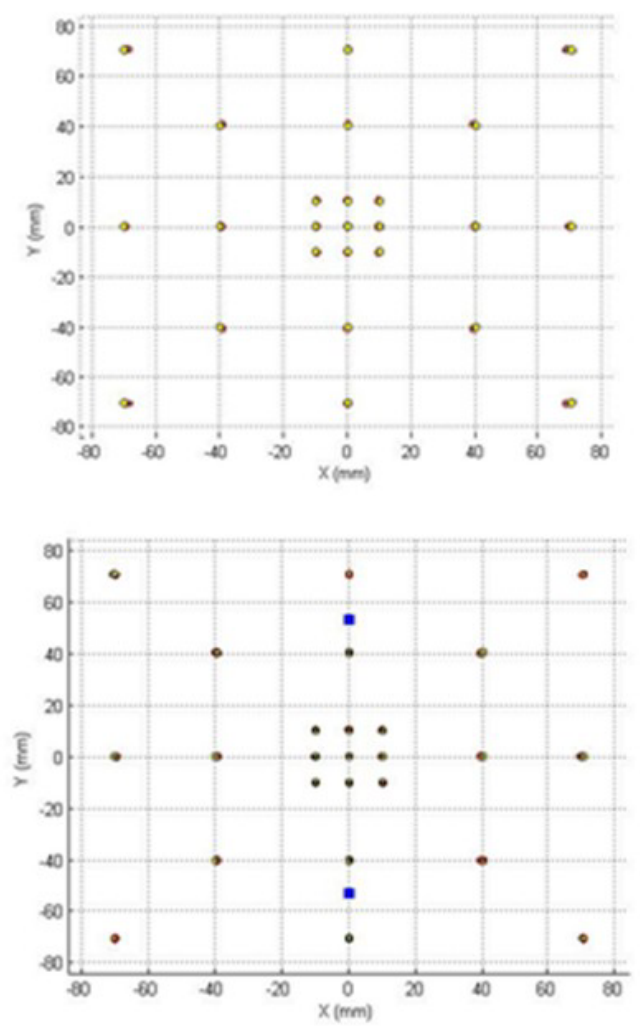

(a)

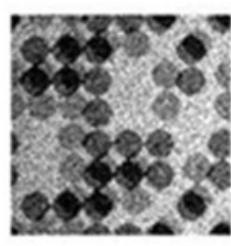

(b)

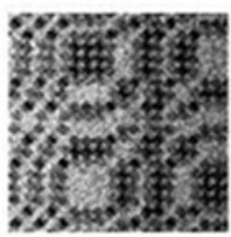

(d)

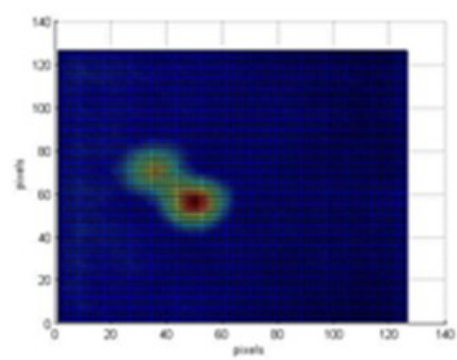

(c)

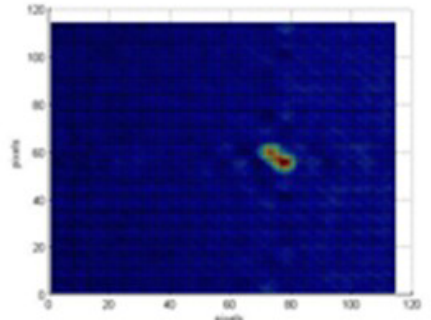

(e)
Figure 5. a) The position estimation (yellow spots) of a ${ }^{241} \mathrm{Am}$ source placed at several locations within the FCFOV $_{S}$ at $\mathrm{Z}=150 \mathrm{~mm}$ for Rank 19 (up) and Rank 7 (down). (b)-(e) Shadowgrams (left) and reconstructed images (right) of two point-like ${ }^{241} \mathrm{Am}$ sources positioned at $(\mathrm{x}, \mathrm{y}, \mathrm{z})=(0,0,150 \mathrm{~mm})$ for Ranks 7 (b, c) and 19 (d, e), respectively. 


\section{References}

1. D. N. Anderson, D. C. Stromswold, S. C. Wunschel, A. J. Peurrung and R. R. Hansen, Technometrics 48, no. 2, p252-261 (2006)

2. J. E. Lees, S.L. Bugby, D. J. Bassford, P. E. Blackshaw and A. C. Perkins, J. Instr. 8, p10021 (2013)

3. Hirofumi Fujii, John D. Idoine, Sylvain Gioux, Roberto Accorsi, David R. Slochower, Richard C. Lanza, John V. Frangioni, Mol. Imag. Biol. 14, p173-182 (2012)

4. Meng L.J., Tan J.W., Spartiotis K., Schulman T., Nucl. Instr. Meth. Phys. Res. A 604, p548 (2009)

5. E.E Fenimore and T. M. Cannon, Applied Optics 20, no. 10, p1858-1864 (1981)

6 A. Gros et al., Astronomy \& Astrophysics 411, p179-183 (2003)

7. I Kaissas, C.Papadimitropoulos, K. Karafasoulis, C. Potiriadis, C.P. Lambropoulos, J. Phys.: Conf. Series 637, 012016 (2015)

8. E.I. Novikova et al., IEEE Nucl. Sci. Symp. Conf. Rec., p607-612 (2006) 